\title{
PROPOSICIÓN CONDICIONAL: INTERPRETACIÓN Y USO POR PARTE DE PROFESORES DE MATEMÁTICAS EN FORMACIÓN
}

\author{
Echeverry, Armando; Molina, Óscar; Samper, Carmen; Perry, Patricia y Camargo, Leonor \\ Universidad Pedagógica Nacional, Bogotá, Colombia \\ aecheverri@pedagogica.edu.co \\ ojmolina@ pedagogica.edu.co \\ csamper@pedagogica.edu.co \\ pperry@yahoo.com.mx \\ lcamargo@pedagogica.edu.co
}

\begin{abstract}
Resumen. Reportamos resultados de una investigación ${ }^{1}$ realizada en torno a la interpretación de la condicional y al uso que de ella hace un grupo de estudiantes para profesor de matemáticas en el marco de un curso de geometría plana. Analizamos los resultados de dos cuestionarios aplicados, uno al inicio del curso y otro al final, con el objetivo de comparar su interpretación y uso de la condicional. Los cambios estarían generados por la implementación, a lo largo del semestre, de algunas estrategias didácticas tendentes a favorecer el acercamiento a los usos e interpretaciones de la condicional desde la matemática.
\end{abstract}

Palabras clave. Demostración, actividad demostrativa, uso e interpretación de afirmaciones condicionales.

\section{Conditional statements: interpretation and use by pre-service mathematics teachers}

Summary. We report research findings with respect to the interpretation of conditional statements and their use by a group of pre-service mathematics teachers of a Euclidean geometry course. We analyze the results of two questionnaires applied to the students, one at the beginning of the course and the other at the end, to compare their interpretation and use of conditionals. The changes would be generated by the implementation of some didactic strategies throughout the semester, to favor the approach to uses and interpretations of the conditional in mathematics.

Keywords. Proof, proofing activity, use and interpretation of conditional statements.

\section{INTRODUCCIÓN}

Nuestro grupo de investigación ${ }^{2}$ ha centrado su labor en aspectos didácticos relacionados con el aprendizaje y la enseñanza de la demostración en geometría. Dicho interés nos condujo hacia el estudio de la interpretación y el uso de la condicional lógica, por la relevancia de ésta en la formulación e interpretación de teoremas y postulados de la geometría, porque su estructura no se adapta naturalmente a la de la expresión «si... entonces...» usada al argumentar en otros ámbitos -siendo aquélla un recurso inicial con el que cuentan los estudiantes cuando comienzan su proceso de aprender a demostrar- y porque es comúnmente aceptado que todo estudiante de mate- máticas debe comprender y construir argumentos matemáticos deductivos, en los cuales la condicional juega un papel decisivo.

Hubo dos razones tras nuestra decisión de profundizar en el tema desde la investigación:

- Son pocos los estudios sistemáticos de las comprensiones que los estudiantes, profesores en formación, tienen de la implicación [condicional] lógica en el contexto de las matemáticas escolares, o de cómo estas comprensiones podrían desarrollarse y cambiar en el transcurso del tiempo. 
- Generalmente, los profesores de matemáticas no somos conscientes de los problemas que tienen los estudiantes al respecto, ni es un tema que se aborde, de forma explícita, en la formación de profesores.

Durante 2006 y 2007 llevamos a cabo una investigación con estudiantes del curso Geometría Plana de la licenciatura en matemáticas de la Universidad Pedagógica Nacional; tuvo como meta identificar actuaciones problemáticas relacionadas con el uso y la interpretación que tienen los estudiantes de la condicional, $\mathrm{y}$ diseñar, poner en marcha y evaluar estrategias que permitieran superarlas (Samper, Perry, Echeverry y Molina, 2008). En este artículo nos centramos en el análisis de los resultados de dos cuestionarios aplicados, uno al inicio del curso y otro al final, con el objetivo de identificar cambios en el uso y la interpretación de la condicional, generados por la implementación de las estrategias didácticas que pusimos en juego. Comenzamos haciendo una síntesis del marco de referencia empleado; después describimos la población y los métodos del estudio desarrollado; a continuación presentamos las categorías con las que analizamos la información de los cuestionarios y los resultados del estudio; finalmente, exponemos una breve discusión de dichos resultados.

\section{MARCO DE REFERENCIA}

Como lo señalan Hoyles y Küchemann (2002), es importante diferenciar los problemas para entender la condicional como objeto matemático de los que tienen que ver con saber usar la condicional para concluir algo. Con respecto a los primeros, estos autores afirman que las experiencias que usualmente tienen los estudiantes los llevan a interpretar la condicional como una proposición hipotética, cuyo enunciado es de la forma «Si $p$, entonces $q »$, que hace referencia exclusivamente al caso en que el antecedente es verdadero. En realidad, bajo esta interpretación se consideran los dos casos en que el antecedente es verdadero, siendo falsa la proposición hipotética si el consecuente es falso. Deloustal-Jorrand (2002) denomina a esta concepción de la condicional concepción causal y señala que induce a los estudiantes a adjudicar carácter de temporalidad, considerando que la situación referida en $p$ debe anteceder en el tiempo a la referida en $q$. Bajo esta concepción, los estudiantes difícilmente pueden entender que la condicional $(p \rightarrow q)$ es verdadera cuando $p$ es una proposición falsa. Su concepción, que es consistente con la lógica natural de la intuición, riñe con la concepción matemática de la condicional, en donde se asume como verdadera una condicional con antecedente falso. ${ }^{3}$

En lo que concierne a los problemas relativos al uso de la condicional para concluir, que dicho sea de paso vemos estrechamente relacionados con los primeros, Laudien (1999) los asocia al uso cotidiano de la condicional y a las experiencias escolares reducidas, en donde los pocos teoremas que se tratan son equivalencias y generalmente se prueba y se usa sólo una de las condicionales involucradas. Esta investigadora encontró evidencia empírica para apoyar la tesis de que los estudiantes usan la condicional si-entonces con el significado de la bicondicional si y sólo si; esto se explica por cuanto, en el uso cotidiano, la condicional está compuesta por dos proposiciones que, tácita o explícitamente, se consideran verdaderas; esto lleva a que tanto la condicional inicial como su recíproca se asuman como afirmaciones verdaderas. Esa concepción errónea de la condicional lleva a dos esquemas de razonamiento no válido que suelen usar los estudiantes y que Laudien denomina negación del antecedente y afirmación del consecuente. El primero se refiere a la situación en que, ante la presentación de una condicional $(p \rightarrow q)$ y la negación del antecedente $(\neg p)$, los estudiantes concluyen la negación del consecuente $(\neg q)$. Los estudiantes, en lugar de reconocer que los datos dados no permiten decidir, desde la lógica matemática, si un objeto tiene la propiedad $q$ cuando no tiene la propiedad $p$, concluyen que no tiene la propiedad enunciada en el consecuente. Por ello, es difícil que los estudiantes entiendan que en la matemática, dadas una condicional y la negación de su antecedente, es igualmente posible que se dé o no el consecuente. El segundo esquema de razonamiento no válido concierne al caso en que, frente a una condicional $(p \rightarrow q)$ y la afirmación de su consecuente $(q)$, al preguntar si el antecedente $(p)$ se puede concluir válidamente, los estudiantes responden afirmativamente, de nuevo desconociendo la imposibilidad de determinar si el antecedente se da o no.

Las respuestas de los estudiantes, producto de los esquemas de razonamiento no válidos, serían adecuadas para las preguntas hechas si se tuviera un enunciado bicondicional. Laudien denomina tales errores como «respuestas implícitas», es decir, esos errores «sugieren implícitamente que se malinterpreta [la condicional] si-entonces como [la bicondicional] si y sólo si». Nuestra hipótesis al respecto consiste en suponer que el razonamiento no válido surge porque no existe en el imaginario de los estudiantes que la combinación antecedente falso y consecuente verdadero determinan una condicional verdadera, desde el punto de vista matemático.

Deloustal-Jorrand (2004) sugiere que la mayoría de las dificultades, tanto en el uso de la condicional como en su comprensión se deben a la falta de establecer vínculos, en el contexto educativo, entre tres perspectivas posibles para dicho concepto: lógica formal, razonamiento deductivo y conjuntos. La primera incluye las tablas de verdad y la cuantificación, y la segunda se refiere al esquema de razonamiento Modus Ponendo Ponens (MPP). La tercera consiste en la interpretación de la tabla de verdad de la condicional por medio de conjuntos. Dada la proposición condicional $p \rightarrow q$, si $P$ es el conjunto de elementos para los cuales el antecedente de la condicional es verdadero y $Q$ está formado por los elementos para los cuales el consecuente es verdadero, se tiene que cada uno de los cuatro conjuntos disyuntos ilustrados por las regiones 1, 2, 3 y 4 en la siguiente figura representa una de las situaciones expresada en las filas de la tabla de verdad. 


$\left.\begin{array}{|c|c|c|c|}\hline & \mathrm{p} & \mathrm{q} & \mathrm{p} \rightarrow \mathrm{q} \\ \hline 1 & \mathrm{~V} & \mathrm{~V} & \mathrm{~V} \\ \hline 2 & \mathrm{~V} & \mathrm{~F} & \mathrm{~F} \\ \hline 3 & \mathrm{~F} & \mathrm{~V} & \mathrm{~V} \\ \hline 4 & \mathrm{~F} & \mathrm{~F} & \mathrm{~V} \\ 1\end{array}\right)$

Tomando conjuntamente los casos 1 y 3 se cumple que las dos proposiciones ( $p \rightarrow q$ y $q$ ) dadas son verdaderas, lo que permite inferir que $p$ puede ser verdadera o falsa. En términos de conjuntos, esto equivale a afirmar que cualquier elemento de $Q$ cumple las dos proposiciones sea que pertenezca o no a $P$, es decir, que cualquier elemento de $(P \cap Q) \cup(Q-P)$ cumple con las condiciones. Cuando las proposiciones dadas corresponden al formato negación del antecedente, es posible identificar que para los casos 3 y 4 se cumplen las dos proposiciones dadas y en ellos $q$ puede ser verdadera o falsa. En términos de conjuntos, esto equivale a afirmar que cualquier elemento de $(Q-P)$ $\cup(P \cup Q)^{\mathrm{C}}$ cumple las dos condiciones.

Estos tres puntos de vista se tuvieron en cuenta para diseñar las categorías mediante las cuales se analizaron las producciones de los estudiantes.

\section{ASPECTOS DEL DISEÑO}

La población de la cual obtuvimos la información estuvo constituida por un grupo de estudiantes del programa de Licenciatura en Matemáticas ${ }^{4}$ inscritos en el curso Geometría Plana en el segundo semestre de 2006. Al principio del semestre diecinueve estudiantes respondieron, durante una sesión de clase, un cuestionario escrito. Durante el semestre, se implementaron cuatro estrategias didácticas que fueron diseñadas con el objetivo de ayudar a superar las actuaciones problemáticas identificadas por el grupo de investigación en torno al uso e interpretación de la condicional. Las actuaciones problemáticas a las que hacemos referencia son: no identificar en forma completa la hipótesis o la tesis de un teorema; aplicar un postulado, teorema o definición sin tener las condiciones que conforman la respectiva hipótesis; concebir de manera restringida la condicional, considerando que el antecedente siempre es verdadero; y considerar que la afirmación y la recíproca o la inversa son equivalentes.

De manera sucinta, las cuatro estrategias didácticas se pueden describir como sigue (más detalles se pueden encontrar en Samper, Perry, Camargo, Molina y Echeverry, sometido a consideración para su publicación):

- Estrategia didáctica A. El propósito es proveer a los estudiantes mecanismos para captar la estructura de una proposición condicional y convertir la información dada en el enunciado de un problema o de una condicional que no está en el formato si-entonces en una fuente confiable. Las acciones que conforman la estrategia son: 1) pedir la identificación completa de la hipótesis y la tesis de los enunciados y la reformulación de éstos en el formato si-entonces cuando sea pertinente hacerlo; 2) exigir la reformulación del enunciado en términos específicos.

- Estrategia didáctica B. El propósito es apoyar a los estudiantes en ver que el uso de la inversa y la recíproca de una proposición condicional verdadera no siempre conduce a un argumento válido, y por qué ello es así. Las acciones que conforman la estrategia son: 1) examinar si el uso de una condicional asociada a una condicional dada conduce a un argumento válido; 2) propiciar la explicitación de parte de los estudiantes del uso que están haciendo de una condicional particular y la comprensión que tienen de ella; 3 ) favorecer el uso de la simbolización lógica de las proposiciones involucradas en un argumento y comparar los esquemas de razonamiento representados con los válidos o analizar sus tablas de verdad.

- Estrategia didáctica $C$. El propósito es proveer experiencias para que los estudiantes comprendan que un enunciado condicional puede hacer referencia a relaciones de dependencia, identifiquen las dos partes que componen un enunciado condicional y discriminen la relación entre el antecedente y el consecuente de un enunciado condicional. Esta estrategia está asociada al trabajo en geometría dinámica. Las acciones que conforman la estrategia son: 1) pedir una descripción de todas las acciones realizadas durante el proceso de construcción y exploración con geometría dinámica; 2) identificar las propiedades construidas y las resultantes.

- Estrategia didáctica $D$. El propósito es proveer elementos heurísticos y técnicos para que los estudiantes puedan emprender por su cuenta procesos deductivos. Esta estrategia está asociada al proceso de deducción requerido para establecer un paso de una demostración. Las acciones que conforman la estrategia son: 1) pedir la identificación de definiciones, postulados y teoremas que tienen la misma tesis que el enunciado que se pretende demostrar o una relación estrecha con ella; 2) analizar por qué se propone hacer una construcción auxiliar dentro de una demostración, identificando claramente la situación que se genera y si los vínculos que se pueden establecer ayudan a avanzar en la elaboración de la demostración; 3) pedir el análisis para determinar si se han establecido todas las condiciones de la hipótesis de un cierto hecho geométrico con miras a usarlo en el proceso deductivo; 4) solicitar la identificación de los pasos previos que en una demostración sustentan cada justificación establecida, con miras a asegurar que es consecuencia lógica de tales pasos.

Al final del semestre, los dieciocho estudiantes que terminaron el curso respondieron otro cuestionario durante una sesión de clase, diseñado para rastrear el progreso en cuanto a la interpretación de la estructura lógica de la condicional y su uso en situaciones que la requerían.

La comparación de las respuestas a los dos cuestionarios nos permitió examinar posibles cambios considerados como efecto de la aplicación de las estrategias didácticas implementadas y hacer algunas reflexiones en torno a la 
superación o no de las actuaciones problemáticas. En ambos cuestionarios, las situaciones propuestas no requerían conocimiento de un contenido especializado - distinto al de la estructura de las proposiciones involucradas y las reglas de inferencia- para poder responder adecuadamente las preguntas; en el diseño de las preguntas tuvimos pre- sente directrices planteadas por investigadores como Watters y English, y Markovits (citados en Laudien, 1999). Además, los cuestionarios fueron diseñados siguiendo el esquema de problemas formulados en Laudien (1999).

El cuestionario inicial fue el siguiente:

Hay dos bolsas diferentes con fichas en las que es posible reconocer tres atributos: color (verde, azul, rojo), forma (redonda, rectangular, triangular) y material con el que está hecha (cartón, plástico). Además, cada ficha de cada bolsa tiene una etiqueta que la identifica (F1, F2, F3, etc.).

A continuación se establecen unas afirmaciones respecto a los atributos de las fichas; a partir de ellas, responda las preguntas, anotando también una explicación para su respuesta. Cada situación se refiere a las fichas de una de las bolsas.

a) Las fichas verdes son triangulares.

Todas las fichas triangulares son del mismo material

\begin{tabular}{|c|c|c|c|c|c|c|}
\hline \multicolumn{2}{|c|}{$\begin{array}{c}\text { INFORMACIÓN SOBRE } \\
\text { FICHAS PARTICULARES }\end{array}$} & PREGUNTA & Sí & NO & NO SE SABE & EXPLICACIÓN \\
\hline i) & F1 es una ficha azul. & ¿Es F1 triangular? & & & & \\
\hline ii) & $\begin{array}{l}\text { F3 y F4 son del mismo } \\
\text { material. }\end{array}$ & $\begin{array}{l}\text { ¿Son F3 y F4 } \\
\text { triangulares? }\end{array}$ & & & & \\
\hline iii) & F5 es redonda. & ¿Es F5 verde? & & & & \\
\hline iv) & F7 y F8 son fichas verdes. & $\begin{array}{l}\text { ¿Son F7 y F8 } \\
\text { del mismo material? }\end{array}$ & & & & \\
\hline
\end{tabular}

b) Si una ficha es de cartón entonces es rectangular.

Si una ficha es de cartón entonces es verde.

\begin{tabular}{|c|c|c|c|c|c|c|}
\hline \multicolumn{2}{|c|}{$\begin{array}{l}\text { INFORMACIÓN SOBRE } \\
\text { FICHAS PARTICULARES }\end{array}$} & PREGUNTA & Sí & NO & NO SE SABE & EXPLICACIÓN \\
\hline i) & F1 es azul. & ¿Es F1 de plástico? & & & & \\
\hline ii) & $\begin{array}{l}\text { F2 es una ficha de } \\
\text { plástico. }\end{array}$ & ¿Es F2 rectangular? & & & & \\
\hline iii) & F3 es rectangular. & $\begin{array}{l}\text { ¿Es F3 una ficha } \\
\text { de cartón? }\end{array}$ & & & & \\
\hline iv) & F5 es de cartón. & ¿Es F5 triangular? & & & & \\
\hline
\end{tabular}

Cuestionario inicial 
El cuestionario final fue:

Un grupo de personas está solicitando visado para viajar a Colombia. Se clasifican según género (masculino o femenino), nacionalidad (italiano, francés, inglés) y raza (blanca, negra, amarilla). Para referirnos a cada persona se le asigna una etiqueta $\left(\mathrm{P}_{1}, \mathrm{P}_{2}, \mathrm{P}_{3} \ldots\right)$. Responda cada pregunta, usando la información dada, y justifique su respuesta de la manera más clara posible.

a) El grupo de personas que fueron el lunes al Consulado tiene las siguientes características:

Las personas de nacionalidad italiana eran de raza blanca.

Todas las personas de raza blanca eran del mismo género.

\begin{tabular}{|l|l|l|l|l|l|l|}
\hline \multicolumn{2}{|c|}{$\begin{array}{c}\text { INFORMACIÓN } \\
\text { SOBRE PERSONAS } \\
\text { PARTICULARES }\end{array}$} & PREGUNTA & Sí & NO & NO SE SABE & EXPLICACIÓN \\
\hline i) & $\mathrm{P}_{1}$ es de raza blanca. & $\begin{array}{l}\text { ¿Es } \mathrm{P}_{1} \text { de nacionalidad } \\
\text { italiana? }\end{array}$ & & & & \\
\hline ii) & $\begin{array}{l}\mathrm{P}_{2} \text { es de nacionalidad } \\
\text { francesa. }\end{array}$ & ¿Es $\mathrm{P}_{2}$ de raza blanca? & & & & \\
\hline iii) & $\begin{array}{l}\mathrm{P}_{3} \text { y } \mathrm{P}_{4} \text { son de } \\
\text { nacionalidad italiana. }\end{array}$ & $\begin{array}{l}\text { ¿Son } \mathrm{P}_{3} \text { y } \mathrm{P}_{4} \text { del } \\
\text { mismo género? }\end{array}$ & & & & \\
\hline iv) & $\begin{array}{l}\mathrm{P}_{5} \text { y } \mathrm{P}_{6} \text { no son } \\
\text { del mismo género. }\end{array}$ & $\begin{array}{l}\text { ¿Son } \mathrm{P}_{5} \text { y } \mathrm{P}_{6} \text { de } \\
\text { nacionalidad francesa? }\end{array}$ & & & & \\
\hline
\end{tabular}

b) Las personas que fueron al Consulado el martes cumplen las siguientes condiciones:

Si es mujer entonces es de nacionalidad inglesa.

Si es mujer entonces es de raza amarilla.

\begin{tabular}{|c|c|c|c|c|c|c|}
\hline \multicolumn{2}{|r|}{$\begin{array}{c}\text { INFORMACIÓN } \\
\text { SOBRE PERSONAS } \\
\text { PARTICULARES }\end{array}$} & PREGUNTA & Sí & NO & NO SE SABE & EXPLICACIÓN \\
\hline i) & $\begin{array}{l}\mathrm{P}_{7} \text { es de nacionalidad } \\
\text { francesa. }\end{array}$ & ¿Es $\mathrm{P}_{7}$ hombre? & & & & \\
\hline ii) & $\mathrm{P}_{8}$ es mujer. & $\begin{array}{l}\text { ¿Es } \mathrm{P}_{8} \text { de raza } \\
\text { amarilla? }\end{array}$ & & & & \\
\hline iii) & $\mathrm{P}_{9}$ es hombre. & $\begin{array}{l}\text { ¿Es } \mathrm{P}_{9} \text { de nacionalidad } \\
\text { francesa? }\end{array}$ & & & & \\
\hline
\end{tabular}


Responder a las preguntas de los cuestionarios exige poner en juego, de manera explícita, esquemas de razonamiento. Referidas a contextos no matemáticos, en ambos casos proveen información por medio de una condicional (afirmación general) y de una proposición simple (afirmación sobre un caso particular) que ha de ser utilizada para responder una pregunta sobre el caso particular. Las decisiones posibles para responder las preguntas formuladas (ocho en el cuestionario inicial y siete en el final) son: Sí, No, o No se sabe. Esta última posibilidad de decisión obedece a una aproximación pragmática a la tarea de preguntar sobre el valor de verdad de una condicional y a la visión de que aprender matemáticas está relacionada con la exploración de objetos para determinar qué propiedades satisfacen y cuáles no. Contestar No se sabe requiere de argumentos que indican cuál es el dominio donde la condicional es verdadera y en cuál es falsa, una acción más exigente que buscar contraejemplos para validar la afirmación (Durand-Guerrier, 2003).

Las afirmaciones generales de los ítems $a$ ) se escribieron dejando implícita la estructura si-entonces de una condicional, y las de los ítems $b$ ) en forma explícita, para comparar el efecto de la formulación del enunciado en las conclusiones obtenidas por los estudiantes. Además, se solicitó a los estudiantes justificar sus decisiones para tener más elementos de juicio en el análisis de las respuestas.

Para clasificar la estructura de la información contenida en cada ítem de los cuestionarios, adaptamos los términos propuestos por Laudien (1999) para caracterizar errores al obtener conclusiones de una inferencia. Específicamente, llamamos formato afirmación del consecuente al conjunto de proposiciones que conforman la información dada en el problema si éstas son una condicional $(p \rightarrow q)$ y la proposición $q$; formato negación del antecedente cuando la información proporcionada consiste en una condicional $(p \rightarrow q)$ y una proposición particular que es negación implícita o explícita del antecedente de la premisa general dada, a la que notamos $(\neg p)$; el formato afirmación del antecedente se refiere al caso cuando se provee una condicional $(p \rightarrow q)$ y la proposición simple es el antecedente $p$, y el formato negación del consecuente se usa para dar cuenta del caso en que la información consiste en una condicional $(p \rightarrow q)$ y la negación del consecuente de la premisa general, sea explícita o implícita, representada por $(\neg q)$.

\section{CATEGORÍAS DE ANÁLISIS}

Las categorías, mediante las cuales analizamos las producciones de los estudiantes, establecen dos tipologías descriptivas que tomadas conjuntamente dan cuenta del uso y de la interpretación de la condicional por parte de los estudiantes. Cabe mencionar que la producción de cada estudiante fue analizada con ambas categorías. Con la categoría tipo de justificación se busca identificar, en el argumento que expone el estudiante para justificar su decisión, cómo usa la información contenida en las dos proposiciones dadas y si el argumento guarda relación con la lógica matemática (p.ej. se fundamenta en un esquema válido de razonamiento o en la tabla de verdad de la condicional desde un punto de vista conjuntista). Por otra parte, en la categoría tipo de condicional se pretende identificar, en el argumento, la interpretación que tiene el estudiante de la condicional sin tener presente la relación que dicho argumento guarda con la decisión que se toma. En ese sentido, no sólo estamos interesados en identificar si los estudiantes usan esquemas de razonamiento o se vislumbra un interés en usarlos, lo cual mostraría una interpretación correcta de la condicional, sino también tratamos de identificar, por ejemplo, si en el argumento se interpreta la condicional como equivalente a su inversa, a su contrarrecíproca o a su recíproca. Específicamente, para analizar las producciones de los estudiantes con respecto a la primera categoría, se tuvo en cuenta el contexto de la situación, mientras que para realizar dicho análisis con relación a la segunda, el énfasis está en la estructura lógica del argumento.

\section{Categoría 1: Tipo de justificación}

Está integrada por cinco elementos para los cuales, además de precisar sus rasgos, presentamos producciones de estudiantes, a manera de ejemplos.

(i) Argumento explícitamente lógico (AEL). La decisión es correcta y podría no estar expresada -como conclusión-dentro de la justificación sino sólo marcada en la casilla dispuesta para tal efecto. La justificación incluye toda la información pertinente proporcionada en el enunciado; además, se representan simbólicamente todas las proposiciones involucradas en la situación que se está analizando, se menciona explícitamente el esquema de razonamiento válido (i.e., Modus Ponendo Ponens (MPP), Modus Tollendo Tollens (MTT), silogismo, equivalencia entre condicional y su contrarrecíproca) en el que se incorporan todas las premisas que intervienen y las conclusiones que se van obteniendo, o se indica, cuando no es posible llegar a una conclusión, que no hay un esquema de razonamiento válido, ya sea representando solamente la información dada o explicitando que no se puede obtener conclusión o aludiendo a la causa por la que no se puede concluir.

\begin{tabular}{|l|l|}
\hline \multicolumn{2}{|l|}{ Situación a (iii) del cuestionario inicial. Las fichas verdes son } \\
triangulares. F5 es redonda. ¿Es F5 verde? \\
\hline \begin{tabular}{l|l} 
Producción de E2 \\
«N
\end{tabular} & Asignación de código \\
«No. Las fichas verdes son de for- & AEL. Decisión correcta. Justifi- \\
ma triangular y F5 es redonda; por & cación incluye las proposiciones \\
lo tanto, no puede ser verde.» & dadas. Se esboza el uso del es- \\
& quema MTT. \\
\hline
\end{tabular}

Ejemplo de asignación del código AEL

Para justificaciones cuyo argumento es explícitamente lógico que no pueden ser clasificadas como AEL porque no se menciona explícitamente toda la información proporcionada, usamos el código AEL*. Nos interesa usar este aspecto como elemento diferenciador porque creemos que no incluir toda la información en su argumento puede ser causa de dificultades, en el futuro, cuando el estudiante tenga que construir una demostración formal. 


\begin{tabular}{|l|l|}
\hline \multicolumn{2}{|l|}{ Situación a (iii) del cuestionario inicial. Las fichas verdes son trian- } \\
gulares. F5 es redonda. ¿Es F5 verde? \\
\hline Producción de E9 & Asignación de código \\
«No. Como F5 es redonda enton- & AEL*. Decisión correcta. Se en- \\
ces F5 no es triangular y como F5 & trevé el uso del esquema MTT, \\
no es triangular entonces F5 no es & pero en la justificación no se men- \\
verde.» & ciona la proposición general. \\
\hline
\end{tabular}

Ejemplo de asignación del código AEL*

(ii) Argumento no completo (ANC). La decisión es correcta. No se evidencia en lo escrito una conexión lógica entre la decisión y la justificación dada; es decir, ni es explícito, ni se entrevé un esquema de razonamiento que pudiera haber sido usado, ya sea natural o formal.

\begin{tabular}{|l|l|}
\hline \multicolumn{2}{|l|}{$\begin{array}{l}\text { Situación a (iii) del cuestionario inicial. Las fichas verdes son trian- } \\
\text { gulares. F5 es redonda. ¿Es F5 verde? }\end{array}$} \\
\hline $\begin{array}{l}\text { Producción de E5 } \\
\text { «No. Por la afirmación de que las } \\
\text { fichas verdes son triangulares.» }\end{array}$ & $\begin{array}{l}\text { Asignación de código } \\
\text { ANC. Decisión correcta. No se } \\
\text { entrevé el uso de algún esquema } \\
\text { que sustente la decisión «No». }\end{array}$ \\
\hline
\end{tabular}

Ejemplo de asignación del código ANC

(iii) Argumento para situación plausible (ASP). Con base en la interpretación de conjuntos, describimos el elemento ASP. La decisión que toma el estudiante no necesariamente es la correcta. Su justificación alude a la posible existencia de contraejemplos, bajo las condiciones dadas, las cuales pueden corresponder al formato negación del antecedente, afirmación del consecuente, o negación de una conjunción. Hace mención al caso antecedente falso, consecuente verdadero; es decir, menciona la existencia de elementos en $(Q-P)$ como contraejemplos. En el caso de la situación negación del antecedente, su respuesta menciona la posible existencia de elementos en $(Q-P)$ y $(P \cup Q)^{\mathrm{C}}$.

\begin{tabular}{|c|c|}
\hline \multicolumn{2}{|c|}{$\begin{array}{l}\text { Situación a (i) del cuestionario inicial. Las fichas verdes son trian- } \\
\text { gulares. F1 es una ficha azul. ¿Es F1 triangular? }\end{array}$} \\
\hline $\begin{array}{l}\text { Producción de E18 } \\
\text { «No se sabe. Ya que no hay la } \\
\text { condición que diga que solamen- } \\
\text { te las fichas verdes son triangula- } \\
\text { res, así fichas de otro color sean } \\
\text { triangulares, entonces f1 puede o } \\
\text { no ser triangular.» }\end{array}$ & $\begin{array}{l}\text { Asignación de código } \\
\text { ASP. La primera parte de la jus- } \\
\text { tificación «no hay la condición } \\
\text { que diga que solamente las fi- } \\
\text { chas verdes son triangulares» } \\
\text { alude a la existencia de elemen- } \\
\text { tos en } Q-P \text {. Además, al afirmar } \\
\text { «así fichas de otro color sean } \\
\text { triangulares, entonces f1 puede o } \\
\text { no ser triangular», creemos que } \\
\text { considera posible la existencia } \\
\text { de elementos en }(P \cup Q)^{\mathrm{C}} \text {. }\end{array}$ \\
\hline
\end{tabular}

Ejemplo de asignación del código ASP

Para justificaciones asociadas a situaciones del formato negación del antecedente, en los que la decisión ha sido correcta pero que no se pueden clasificar como ASP, por- que no se hace alusión a la posible existencia de elementos en $(P \cup Q)^{\mathrm{C}}$, se asigna el código ASP*.

\begin{tabular}{|l|l|}
\hline \multicolumn{2}{|l|}{$\begin{array}{l}\text { Situación a (i) del cuestionario inicial. Las fichas verdes son trian- } \\
\text { gulares. F1 es una ficha azul. ¿Es F1 triangular? }\end{array}$} \\
\hline $\begin{array}{l}\text { Producción de E19 } \\
\text { «No se sabe. Al indicarnos que }\end{array}$ & $\begin{array}{l}\text { Asignación de código } \\
\text { ASP*. La justificación se enfoca }\end{array}$ \\
las fichas verdes son triángulos, & a la existencia de fichas triangula- \\
se puede deducir que no necesa- & res no verdes, es decir, alude a la \\
riamente todas las fichas trian- & existencia de elementos en $Q-P$ \\
gulares son verdes, puede haber & pero no en $(P \cup Q)^{\mathrm{C}}$. \\
más fichas triangulares de dife- \\
rentes colores.»
\end{tabular}

Ejemplo de asignación del código ASP*

(iv) Argumenta falta de información (AFI). La decisión puede ser o no correcta. Este tipo de justificación alude a la falta de información. En algunos casos parece que el estudiante escoge la decisión No se sabe como consecuencia del análisis que ha hecho de la situación desde el punto de vista de la lógica natural, donde no se puede tomar decisiones cuando no está completa la información. Por otro lado, algunos estudiantes parecen estar pensando Personalmente, no puedo decidir porque no tengo el conocimiento necesario para ello, en vez de Es la lógica la que me asegura que no se puede decidir. Para ejemplificar una respuesta en la cual es a partir de la lógica que decide No se sabe, se transcribe en el siguiente cuadro la respuesta del estudiante E16. Para el caso en el cual toma la decisión No se sabe, porque se siente incapaz de tomar una decisión, se presenta como ejemplo la justificación del estudiante E3.

\begin{tabular}{|l|l|}
\hline $\begin{array}{l}\text { 1) Situación a (i) del cuestionario inicial. Las fichas verdes son } \\
\text { triangulares; F1 es una ficha azul. ¿Es F1 triangular? }\end{array}$ \\
\hline $\begin{array}{l}\text { Producción de E16 } \\
\text { «Como todas las fichas verdes } \\
\text { son triangulares, una azul no se } \\
\text { sabe qué forma tenga.» }\end{array}$ & $\begin{array}{l}\text { Asignación de código } \\
\text { AFI. Es a partir de la lógica como } \\
\text { toma la decisión cuando se en- } \\
\text { foca en la necesidad explícita de } \\
\text { conocer la forma de las fichas } \\
\text { azules. }\end{array}$ \\
\hline
\end{tabular}

2) Situación b (i) del cuestionario inicial. Si una ficha es de cartón entonces es verde. F1 es azul. ¿Es F1 de plástico?

\section{Producción de E3}

«No se sabe, puesto que no haAFI. La justificación no tiene blan de las fichas con color azul y condicional dada para obtener a F1 se le puede asignar o plástico una conclusión; en cambio, aluo cartón.»

de a la falta de una condicional relativa al material de las fichas azules.

Ejemplos de asignación del código AFI

(v) Argumento no pertinente (ANP). La decisión puede ser correcta o no. La justificación no guarda relación con la lógica matemática o con la información proporcionada en el enunciado de la pregunta; se puede usar toda o parte de la información proporcionada. 


\begin{tabular}{|l|l|}
\hline $\begin{array}{l}\text { Situación a (i) del cuestionario inicial. Las fichas verdes son trian- } \\
\text { gulares. F1 es una ficha azul. ¿Es F1 triangular? }\end{array}$ \\
\hline $\begin{array}{l}\text { Producción de E15 } \\
\text { «No. Porque la afirmación es- } \\
\text { pecifica que sólo las verdes son } \\
\text { triangulares. }\end{array}$ & $\begin{array}{l}\text { Asignación de código } \\
\text { ANP. La justificación destaca que } \\
\text { no hay fichas triangulares que no } \\
\text { sean verdes, con lo cual se está } \\
\text { invirtiendo el sentido de la con- } \\
\text { dicional dada, es decir, se estaría } \\
\text { tomando la recíproca en vez de la } \\
\text { condicional dada, situación que no } \\
\text { es coherente con la lógica formal. }\end{array}$ \\
\hline
\end{tabular}

Ejemplo de asignación del código ANP

\section{Categoría 2: Uso de la condicional dada o alusión a una condicional asociada}

La segunda categorización concierne más puntualmente a la condicional como elemento de la justificación con la que el estudiante explica su decisión. Atiende al uso evidente de los esquemas de razonamiento MPP o MTT o de una supuesta condicional que le permitiría usar alguno de esos esquemas para tomar una decisión. Se entrevé la búsqueda de elementos necesarios para poder usar MPP o MTT, o el uso, incorrecto, de la recíproca o inversa de alguna de las afirmaciones dadas como información. En lo que sigue, $p$ representa el antecedente de alguna afirmación dada como dato, y $q$ representa al consecuente.

(i) Condicional donde el consecuente de una de las afirmaciones que se presentan como datos se convierte en antecedente de una condicional cuyo consecuente es otra propiedad (CA). El estudiante alude a no tener una proposición de la forma $(q \rightarrow x)$, donde $x$ es alguna propiedad relacionada con la pregunta que se hace, o usa la recíproca como si fuera equivalente a la afirmación original.

Situación a (ii) del cuestionario inicial. Todas las fichas triangulares son del mismo material; F3 y F4 son del mismo material. ¿Son F3 y F4 triangulares?

\section{Producción de E4}

«Sí. Porque si la afirmación nos dice que «todas las fichas triangulares son del mismo material» y si F3 y F4 son del mismo material, entonces $\mathrm{F} 3$ y $\mathrm{F} 4$ son triangulares.»

\section{Asignación de código}

CA. Utiliza la recíproca de la premisa general como si fuera equivalente a ésta.

Situación a (i) del cuestionario inicial. Las fichas verdes son triangulares. F1 es una ficha azul. ¿Es F1 triangular?

\section{Producción de E8}

«No se sabe. Ya que la afirmación me dice que las fichas verdes son triangulares, mas puede existir la posibilidad de que haya fichas triangulares de otro color.»

Asignación de código
CA. Al decir puede existir la
posibilidad de que haya fichas
triangulares de otro color, se está
refiriendo a que una afirmación de
la forma «Las fichas triangulares
son...», permitiría contestar «Sí»
o «No» a la pregunta.

Ejemplos de asignación del código CA en la situación de formato de negación del antecedente

(ii) Condicional donde el antecedente está relacionado con la negación del antecedente de alguno de los datos presentados y el consecuente es otra propiedad (NA). El estudiante menciona no tener una proposición de la forma $(r \rightarrow x)$, donde $r$ es equivalente a $(\neg p)$ o es parte de la negación de $p, \mathrm{y} x$ es alguna propiedad relacionada con la pregunta que se hace, o usa la inversa porque considera que es equivalente a la proposición dada.

\begin{tabular}{|l|l|}
\hline \multicolumn{2}{|l|}{$\begin{array}{l}\text { Situación a (i) del cuestionario inicial. Las fichas verdes son trian- } \\
\text { gulares. F1 es una ficha azul. ¿Es F1 triangular? }\end{array}$} \\
\hline $\begin{array}{l}\text { Producción de E9 } \\
\text { «No. Como F1 es una ficha azul, } \\
\text { entonces no es verde y como F1 no } \\
\text { es verde, entonces no es triangular.» }\end{array}$ & $\begin{array}{l}\text { Asignación de código } \\
\text { NA. Específicamente, él usa como } \\
\text { válida la inversa de la proposición } \\
\text { original. }\end{array}$ \\
\hline $\begin{array}{l}\text { Situación b (i) del cuestionario inicial. Si una ficha es de cartón } \\
\text { entonces es verde. F1 es azul. ¿Es F1 de plástico? }\end{array}$ \\
\hline $\begin{array}{l}\text { Producción de E13 } \\
\text { «F1 es azul, pero aún no sabemos } \\
\text { cuáles son las características de } \\
\text { una ficha de plástico.» }\end{array}$ & $\begin{array}{l}\text { Asignación de código } \\
\text { NA. Ahí, alude a la necesidad de } \\
\text { tener una condicional en la cual el } \\
\text { antecedente es la negación del an- } \\
\text { tecedente de la condicional dada, } \\
\text { y la información proporcionada } \\
\text { corresponde al caso negación del } \\
\text { consecuente. }\end{array}$ \\
\hline
\end{tabular}

Ejemplos de asignación del código NA en las dos situaciones descritas

(iii) Condicional donde el antecedente está relacionado con la negación del consecuente de alguno de los datos dados y el consecuente es otra propiedad (NC). El estudiante hace referencia a no tener una proposición como $(s \rightarrow x)$ donde $s$ equivale a $(\neg q)$ o es parte de la negación de $q$ y $x$ es alguna propiedad relacionada con la pregunta que deben responder, usa la contrarrecíproca o se entrevé una demostración por contradicción.

Situación a (iii) del cuestionario inicial. Las fichas verdes son triangulares. F5 es redonda. ¿Es F5 verde?

\begin{tabular}{|l|l|}
\hline Producción de E11 & Asignación de código \\
«No se sabe. Porque no cono- & NC. Con ello, expresa que hace \\
cemos las características de las & falta una condicional en la cual ser \\
fichas redondas.» & ficha redonda sea el antecedente. \\
\hline
\end{tabular}

Ejemplo de asignación del código $\mathrm{NC}$

(iv) No equivalencia de la recíproca y la condicional dada (NR). El estudiante enuncia explícitamente que una condicional y su recíproca no son equivalentes.

Situación a (i) del cuestionario inicial. Las fichas verdes son triangulares. F1 es azul. ¿Es F1 triangular?

\begin{tabular}{l|l}
\hline $\begin{array}{l}\text { Producción de E10 } \\
\text { «No se sabe. Las fichas triangulares }\end{array}$ & Asignación de código \\
NR.
\end{tabular}

son de color verde, pero también pueden ser de otro color ya que la afirmación nos dice que todas las fichas verdes son triangulares, mas no dice que todas las fichas triangulares son de color verde.»

Ejemplo de asignación del código NR 
(v) Esquema válido (EV). La justificación muestra el uso de un esquema de razonamiento válido, sin que el estudiante necesariamente haga explícito todo el proceso de razonamiento, o lo menciona, o hace referencia a los valores de verdad de la proposición, o indica que no hay esquema válido para concluir algo.

\begin{tabular}{l} 
Situación a (iii) del cuestionario inicial. Las fichas verdes son \\
triangulares. F5 es redonda. ¿Es F5 verde? \\
\hline \begin{tabular}{l|l|} 
Producción de E9 & Asignación de código \\
«Como F5 es redonda entonces & EV. En ella se reconoce el uso \\
F5 no es triangular y como F5 & del esquema de razonamiento \\
no es triangular entonces F5 no & MTT. \\
es verde.» & \\
\hline
\end{tabular}
\end{tabular}

Ejemplo de asignación del código EV

$(\mathbf{E V} *)$ : Tras haber usado un esquema válido, el estudiante menciona una proposición que no tiene que ver con éste o con la conclusión obtenida, y que es incorrecta.

Situación a (iii) del cuestionario inicial. Las fichas verdes son triangulares. F5 es redonda. ¿Es F5 verde?

\begin{tabular}{l|l|}
\hline Producción de E19 & Asignación de código \\
«Se nos indica primero que las & $\mathrm{EV}^{*}$. \\
fichas verdes son triangulares, & \\
y si la forma triangular ya está & \\
definida para el color verde en- & \\
tonces las fichas redondas no \\
pueden ser verdes. Aunque en \\
la afirmación inicial no dicen \\
que todas las fichas verdes son \\
triangulares.»
\end{tabular}

Ejemplo de asignación del código $\mathbf{E V *}$

(vi) Información dada (ID). El estudiante menciona solamente la condicional dada en la información general o su contrarrecíproca, sin otras frases que lleven a inferir el razonamiento que realizó, o repite esa misma información en forma de justificación.

Situación a (iii) del cuestionario inicial. Las fichas verdes son triangulares. F5 es redonda. ¿Es F5 verde?

Producción de E3
«No, porque las fichas verdes
son triangulares».

\section{Asignación de código}

ID. La justificación dada involucra sólo la información dada en el enunciado.

Ejemplo de asignación del código ID

(vii) Fuera de contexto (FC). Se asigna este elemento cuando no hay justificación, el estudiante menciona una condicional que no es equivalente con la información dada, incluye información que no está relacionada con la dada, menciona la necesidad de tener afirmaciones de dos de los tipos de condicional definidas en esta categorización, cuando no hay condicional mencionada o cuando escribe un esquema con premisas que no corresponde a uno válido.
Situación b (i) del cuestionario inicial. Si una ficha es de cartón entonces es verde. F1 es azul. ¿Es F1 de plástico?

\section{\begin{tabular}{l|l} 
Producción de E8 & Asignación de código
\end{tabular}}

«Porque no poseemos más infor- FC. Se entrevé el deseo de tener mación acerca de las fichas que una condicional cuyo antecedenson plásticas ni azules».

te sea la negación del anteceden-

te de la premisa general dada $y$, a

la vez, una condicional que tenga como antecedente la negación del consecuente de esa premisa.

Ejemplo de asignación del código FC

Para finalizar esta sección, con el propósito de ilustrar cómo las dos categorías proporcionan información diferente, se presentan los siguientes ejemplos.

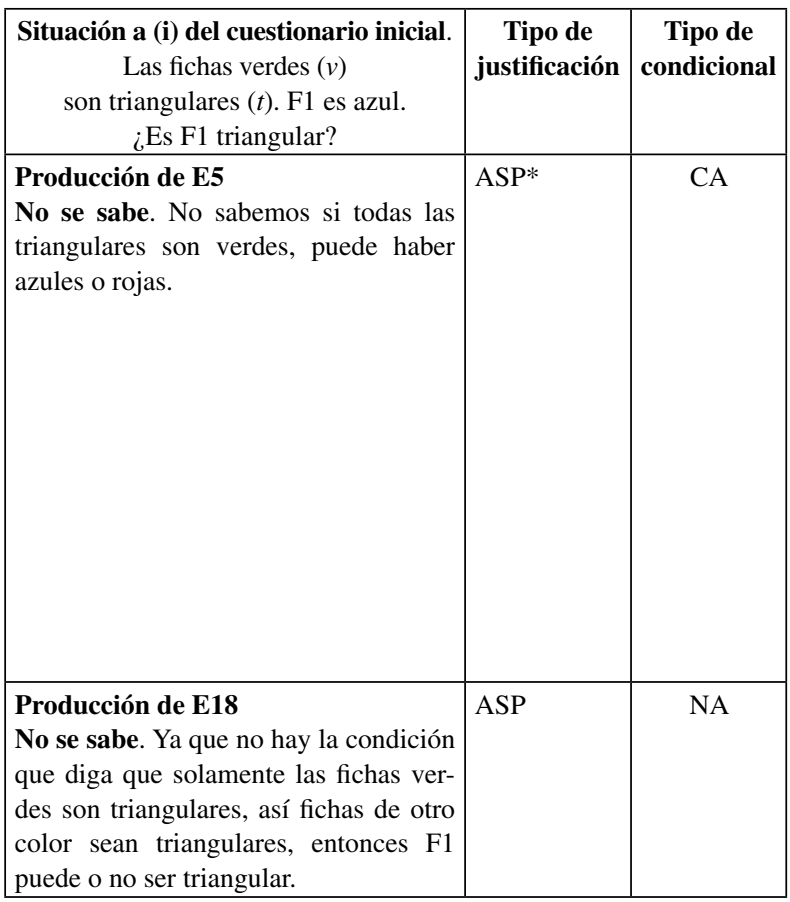

En cuanto al tipo de condicional, identificamos el elemento CA en el argumento del estudiante E5, puesto que éste se fundamenta en que no se tiene la validez de la condicional $t \rightarrow v$. En su argumento, E18 menciona «fichas de otro color», la negación del antecedente de la condicional dada, aludiendo a que no se tiene la validez de la inversa de la implicación dada, razón por la cual fue clasificada con el elemento NA. Por ende la interpretación de la condicional de ambos estudiantes es correcta, pues reconocen que una condicional no es lógicamente equivalente a su recíproca ni a su inversa.

En relación con el tipo de justificación, como E5 reconoce la no validez de la recíproca, está aceptando la posibilidad de tener una ficha que cumpla $t \wedge \neg v$. En su argumento, le falta aludir a la posible existencia de una ficha azul no triangular $\left((P \cup Q)^{\mathrm{C}}\right)$, en contraposición a lo que sí hace E18, factor que hace que su justificación 
para escoger como respuesta «No se sabe» no sea completa. Por ello, el argumento de E5 es ASP* y el de E18 ASP. En este caso, se determina que la comprensión que tiene E5 de la condicional no se corresponde con lo que ella es desde la matemática.

\section{ELEMENTOS PARA INTERPRETAR LAS PRO- DUCCIONES DE LOS ESTUDIANTES}

Describiremos a continuación los elementos que consideramos deseable encontrar en las respuestas de los estudiantes para hacer un contraste entre los resultados de los cuestionarios inicial y final, de tal forma que nos permita hacer algunas inferencias acerca de los resultados de la aplicación de las estrategias a lo largo del curso.

En lo que concierne al tipo de justificación, lo que consideramos deseable no está supeditado a una decisión correcta, pues nuestro principal interés está en el discurso mismo que plasma el estudiante para justificar su decisión.

Cuando se proponen situaciones correspondientes a los formatos negación del antecedente o afirmación del consecuente son igualmente aceptables justificaciones del tipo AEL-AEL*, o ASP - ASP*. Las justificaciones del primer tipo, porque con ellas se estaría reconociendo que no hay un esquema de razonamiento válido que pueda aplicarse a la información dada para deducir algo que sea verdadero. Las del segundo tipo, porque se estaría reconociendo que pueden existir varias posibilidades de conclusión para las cuales la información dada es verdadera.

En cuanto a las situaciones correspondientes a los formatos afirmación del antecedente y negación del consecuente, el único tipo de justificación aceptable es AEL o AEL*, ya que se estaría reconociendo que la información proporcionada corresponde al conjunto de proposiciones que permite aplicar un esquema de razonamiento válido: MPP para el primero y MTT para el segundo. Pero en el ítem $a(i v)$ del cuestionario final, debido a las características especiales de la situación -que se expondrán más adelante- también son deseables justificaciones del tipo ASP o ASP*.

En esta categoría no se considera deseable la justificación de tipo ANC, puesto que la decisión tomada no es consecuencia lógica del argumento presentado. Tampoco es deseable un argumento clasificado como AFI, ya que los estudiantes recurren a la lógica natural para tomar su decisión o expresan falta de conocimiento para dar como respuesta Sí o No. Finalmente, aquellos argumentos de tipo ANP no son deseables, puesto que en ellos se utiliza información diferente a la proporcionada o no se procede de acuerdo con la lógica formal.

En lo que concierne al tipo de condicional, para cada formato que corresponde al conjunto de proposiciones que conforman la información, determinamos cuáles de los elementos de esta categoría son los deseables atendiendo a la concordancia entre la decisión correcta y la justificación, porque es la conjugación de ellas lo que permite identificar el uso adecuado de la condicional.
Con respecto a esta categoría, es deseable que las situaciones con formato afirmación del antecedente o negación del consecuente queden categorizadas como EV o $\mathrm{EV}^{*}$, puesto que esto podría indicar que los estudiantes reconocen que se puede utilizar el esquema MPP o MTT. En las situaciones de formato afirmación del consecuente, cuando la decisión es correcta, son esperados los elementos $\mathrm{EV}, \mathrm{EV}^{*}$, NR o CA. En los tres primeros casos podría haber evidencia de que el estudiante reconoce que no hay una forma válida desde la lógica para tomar una decisión distinta a No se sabe. El último elemento podría indicar que el estudiante reconoce la ausencia de información específica que permita tomar la decisión Sí o No, o establece la posibilidad de la existencia de contraejemplos. A pesar de que en una decisión correcta se puede presentar el elemento NA, no es esto lo deseable porque podría indicar que el estudiante no reconoce que no hay un esquema de razonamiento que permita, a partir de lo que se tiene y se quiere, concluir datos que sirvan para responder la pregunta. En las situaciones de formato negación del antecedente, cuando la decisión es correcta, son deseables los elementos EV, EV*, NR o NA; para los tres primeros valores, las razones son las mismas expuestas anteriormente; para el caso NA, la explicación es la que se dio para CA, en el formato afirmación del consecuente. Asimismo, puede presentarse, en una decisión correcta, el elemento CA, pero esto no es lo deseable porque no se entrevé que el estudiante reconozca que ningún esquema de razonamiento válido permite deducir, a partir de los datos que se tienen y lo que el estudiante quiere, algún dato que sirva para contestar la pregunta.

En esta categoría no se consideran deseables los elementos ID y FC; el primero porque al presentar como justificación sólo la condicional dada como información no se puede identificar el uso que a ésta se le da para tomar su decisión; y el segundo porque no se puede determinar en el argumento, si lo hay, cómo se usó la condicional o porque el uso de ésta no se corresponde con los lógicamente válido.

\section{RESULTADOS DEL ESTUDIO}

Para presentar los resultados, en las siguientes tablas reportamos el número de estudiantes que dieron una justificación con elementos deseables para cada situación según el formato respectivo; ahora bien, para obtener los porcentajes que se reportan en las tablas, se comparó el número total de producciones relativas a las situaciones de cada uno de los formatos con el número de producciones clasificadas como deseables para las situaciones correspondientes. Este último número varía porque diecinueve estudiantes respondieron el cuestionario inicial y dieciocho el final; como se analizan todas las situaciones que le corresponden a cada formato, que pueden ser una o dos, el número total de respuestas posibles es 38 para el primer caso y 18 o 36 para el segundo. Además, para complementar el análisis se tuvieron en cuenta el número de decisiones correctas. Como las dos categorías se complementan y dan información respecto al uso y comprensión de la condicional, en el análisis se interrelacionan los resultados de cada categoría. 


\section{En cuanto al formato negación del antecedente}

Cuando la información de la situación corresponde al formato negación del antecedente, la decisión correcta es No se sabe. En los ítems que corresponden a este formato, la negación del antecedente no es explícita salvo en el caso del ítem $b$ (iii) del cuestionario final; en los otros tres $(a(i)$ y $b$ (ii) del cuestionario inicial; $a$ (ii) del cuestionario final), la negación es implícita, pues resulta a partir del principio del tercio excluido (si es $A$, no es $B$ ). Para estos casos, si es azul no es verde, si es de plástico no es de cartón, y si es de nacionalidad francesa no es de nacionalidad italiana. El esquema lógico que representan estas situaciones es $[(p \rightarrow q) \wedge \neg p] \rightarrow \bullet^{6}$.

En la tabla 1 se consignan los resultados cuantitativos.

El número de decisiones correctas en el cuestionario final (19 de 38 o $50 \%$ ) mejoró ostensiblemente con respecto al cuestionario inicial (30 de 36 o 83,33\%). Esta evidencia, junto con el hecho de que los números relacionados con el tipo de justificación deseable hayan disminuido pero haya aumentado el número de argumentos clasificados como NA, nos indica: (i) mayor comprensión acerca de la validez de esquemas de razonamiento, y (ii) menos personas que centran su justificación en ese hecho pero más personas que reconocen la información que necesitarían para poder hacer una deducción desde la lógica usando MPP. Además, si nos fijamos en los resultados de la categoría tipo de condicional, la forma en la que se presenta la negación del antecedente no parece ser un factor que influya seriamente en el reconocimiento del formato lógico de la información presentada, ya que en el cuestionario final se evidencia que la diferencia en las frecuencias relativas, cuando la negación es implícita a cuando es explícita, es mínima

\section{En cuanto al formato afirmación del consecuente}

El esquema simbólico que se expresa en las situaciones $a$ (ii) y $b$ (iii) del cuestionario inicial, y en $a(i)$ del cuestionario final es $[(p \rightarrow q) \wedge q] \rightarrow \bullet$, lo cual no se corresponde con un esquema de razonamiento válido. Esto significa que la decisión correcta a tomar también es $N o$ se sabe. A diferencia del formato negación del antecedente, en este caso la afirmación del consecuente tiene que ser explícita.

En la tabla 2 se consignan los resultados cuantitativos.

\begin{tabular}{|c|c|c|c|c|c|}
\hline \multirow{2}{*}{\multicolumn{2}{|c|}{$\begin{array}{c}\text { CATEGORÍA Y ELEMENTOS } \\
\text { DESEABLES }\end{array}$}} & \multicolumn{2}{|c|}{ CUESTIONARIO INICIAL } & \multicolumn{2}{|c|}{ CUESTIONARIO FINAL } \\
\hline & & a (i) & $\mathrm{b}$ (ii) & a (ii) & b (iii) \\
\hline \multirow{4}{*}{ Tipo de justificación } & AEL & 1 & 1 & 2 & 3 \\
\hline & \begin{tabular}{|l|l} 
AEL* $^{*}$ \\
\end{tabular} & 3 & 3 & 2 & 0 \\
\hline & ASP & 1 & 0 & 0 & 1 \\
\hline & ASP* & 6 & 1 & 2 & 0 \\
\hline \multicolumn{2}{|c|}{ Total } & \multicolumn{2}{|c|}{16 de $38(42,10 \%)$} & \multicolumn{2}{|c|}{10 de $36(27,77 \%)$} \\
\hline \multirow{4}{*}{ Tipo de condicional } & EV & 0 & 0 & 0 & 1 \\
\hline & $\mathrm{EV}^{*}$ & 0 & 0 & 0 & 0 \\
\hline & NR & 5 & 4 & 3 & 1 \\
\hline & NA & 1 & 3 & 6 & 5 \\
\hline \multicolumn{2}{|c|}{ Total } & \multicolumn{2}{|c|}{13 de $38(34,21 \%)$} & \multicolumn{2}{|c|}{16 de $36(44,44 \%)$} \\
\hline
\end{tabular}

Frecuencias de justificaciones de los alumnos, en los elementos deseables de las dos categorías, para situaciones del formato negación del antecedente.

Tabla 2

\begin{tabular}{|c|c|c|c|c|}
\hline \multirow{2}{*}{\multicolumn{2}{|c|}{$\begin{array}{c}\text { CATEGORÍA Y ELEMENTOS } \\
\text { DESEABLES }\end{array}$}} & \multicolumn{2}{|c|}{ CUESTIONARIO INICIAL } & \multirow{3}{*}{$\begin{array}{c}\text { CUESTIONARIO FINAL } \\
\mathrm{a}(\mathrm{i}) \\
\end{array}$} \\
\hline & & a (ii) & $\mathrm{b}$ (iii) & \\
\hline \multirow{4}{*}{ Tipo de justificación } & AEL & 1 & 0 & \\
\hline & AEL* & 3 & 1 & 7 \\
\hline & ASP & 5 & 4 & 2 \\
\hline & ASP* & 0 & 0 & 0 \\
\hline \multicolumn{2}{|r|}{ Total } & \multicolumn{2}{|c|}{14 de $38(36,84 \%)$} & 11 de $18(61,11 \%)$ \\
\hline \multirow{5}{*}{ Tipo de condicional } & EV & 0 & 0 & 1 \\
\hline & $\mathrm{EV}^{*}$ & 0 & 0 & 0 \\
\hline & NR & 3 & 3 & 7 \\
\hline & $\mathrm{CA}$ & 3 & 5 & 1 \\
\hline & Total & \multicolumn{2}{|c|}{14 de $38(36,84 \%)$} & 9 de $18(50 \%)$ \\
\hline
\end{tabular}

Frecuencias de justificaciones de los alumnos, en los elementos deseables de las dos categorías, para situaciones del formato afirmación del consecuente. 
De manera global, en el cuestionario final es mayor el porcentaje de decisiones correctas (14 aciertos de 18 o $77,77 \%$ ) con respecto al del cuestionario inicial (23 aciertos de 38 o $60,52 \%$ ). La diferencia entre los resultados obtenidos en el ítem $b$ (iii) del cuestionario inicial y los logrados en los otros dos ítems es notoria. Esto se puede deber a la diferencia entre la estructura de la premisa general, pues en los ítems $a$ (ii) y $a(i)$ se usa el cuantificador universal y en $b$ (iii) no. Además, es notorio el aumento de la cantidad de elementos que hemos catalogado como deseables en ambas categorías. Cabe destacar que en los resultados del cuestionario inicial, la diferencia en la forma en que los estudiantes usaron la condicional en términos de NR y CA no es significativa (6 y 8, respectivamente); mientras que en el cuestionario final, el uso de la condicional por parte de ellos se concentró en la no equivalencia de la condicional con su recíproca, es decir, en el elemento NR. Este resultado es una evidencia positiva del efecto de una estrategia que consistió en identificar la estructura de la información que se tiene para poder establecer una conclusión válida, la cual fue enfatizada de manera especial a lo largo del desarrollo del curso.

\section{En cuanto al formato afirmación del antecedente}

Cuando la información se presenta por medio de premisas que son una condicional y su antecedente ( $b$ (iv) del cuestionario inicial y $b$ (ii) del cuestionario final), el uso del esquema MPP permite deducir información veraz, útil para responder la pregunta ${ }^{7}$. Las situaciones de los dos cuestionarios difieren, pues en $b$ (ii) se deduce directamente la decisión, mientras que en $b$ (iv) no, ya que en esta situación, además de usar tal esquema, se debe usar el principio del tercio excluido (se debe tener en cuenta que una ficha no puede tener dos formas al mismo tiempo $)^{8}$, o hacer un razonamiento por contradicción (suponiendo que la ficha no fuese rectangular) ${ }^{9}$.

En los dos cuestionarios se incluyeron situaciones que, aun cuando corresponden al formato afirmación del antecedente, pueden ser abordadas de forma diferente $(a$ (iv) del cuestionario inicial y a (iii) del cuestionario final). En ellas se puede aplicar el silogismo

$[(p \rightarrow q) \wedge(q \rightarrow r)] \rightarrow(p \rightarrow r) ;[(p \rightarrow r) \wedge p] \rightarrow r$ o aplicar dos veces MPP

$[(p \rightarrow q) \wedge p] \rightarrow q ;[(q \rightarrow r) \wedge q] \rightarrow r$

obteniendo en ambos casos directamente la decisión.

En la tabla 3 se presentan los resultados numéricos.

Los resultados globales no parecen verse afectados por la diferencia entre las dos formas argumentales, pues tanto en el cuestionario inicial como en la final todos los estudiantes toman la decisión correcta cuando el esquema es silogismo. En el cuestionario inicial 35 de los 38 estudiantes $(92,10 \%)$ responde correctamente; es entendible que tres estudiantes tomen una decisión incorrecta en el ítem $b$ (iv) porque requería usar otro esquema además de MPP. Sorprendentemente, después de la implementación de las estrategias no todos los estudiantes mostraron en sus justificaciones el uso explícito del esquema MPP como lo esperábamos, aunque todos los estudiantes respondieron correctamente en el cuestionario final. Para las situaciones de este formato es difícil elaborar una justificación para una decisión que para los estudiantes es evidente y probablemente por eso sólo repiten la información dada no pudiendo ser categorizadas sus producciones con el elemento EV. Un análisis global muestra que, cuando la información corresponde al formato afirmación del antecedente, es más frecuente la decisión correcta.

\section{En cuanto al formato negación del consecuente}

Cuando la información corresponde a este formato, el esquema de razonamiento aplicado debe ser MTT para obtener datos que permitan contestar la pregunta. En $a$ (iii) del cuestionario inicial la decisión se toma de manera directa al utilizar el esquema, ya que la pregunta se refiere a la hipótesis de la condicional. Esta situación difiere de las otras tres $(b(i)$ del cuestionario inicial; $b(i)$ y $a$ (iv) del cuestionario final), ya que en éstas, además de tener que utilizar MTT, debe hacerse uso de Modus Tollendo Ponens (MTP), pues se pregunta sobre la característica de la ficha o persona en términos de la negación del antecedente de la condicional, que para estos casos tiene solamente dos opciones (plástico o cartón en el cuestionario inicial, y hombre o mujer, en la ta-

Tabla 3

\begin{tabular}{|c|c|c|c|c|c|}
\hline \multirow{2}{*}{\multicolumn{2}{|c|}{ CATEGORÍA Y VALORES DESEABLES }} & \multicolumn{2}{|c|}{ CUESTIONARIO INICIAL } & \multicolumn{2}{|c|}{ CUESTIONARIO FINAL } \\
\hline & & a (iv) & $\mathrm{b}$ (iv) & a (iii) & $\mathrm{b}$ (ii) \\
\hline \multirow{2}{*}{ Tipo de justificación } & AEL & 2 & 0 & 0 & 3 \\
\hline & AEL* & 7 & 14 & 11 & 8 \\
\hline \multicolumn{2}{|r|}{ Total } & \multicolumn{2}{|c|}{23 de $38(60,52 \%)$} & \multicolumn{2}{|c|}{22 de $36(61,11 \%)$} \\
\hline \multirow{2}{*}{ Tipo de condicional } & EV & 14 & 14 & 13 & 7 \\
\hline & $\mathrm{EV}^{*}$ & 0 & 0 & 0 & 0 \\
\hline \multicolumn{2}{|r|}{ Total } & \multicolumn{2}{|c|}{28 de $38(73,68 \%)$} & \multicolumn{2}{|c|}{20 de $36(55,55 \%)$} \\
\hline
\end{tabular}

Frecuencias de justificaciones de los alumnos, en los elementos deseables de las dos categorías, para situaciones del formato afirmación del antecedente. 
rea final). Por ello, en estas tres situaciones la decisión se toma indirectamente. Por otro lado, $a$ (iv) difiere de las demás porque la premisa particular es la negación explícita del consecuente de una de las premisas generales. En este caso, se debe aplicar dos veces el esquema MTT y estudiar o no dos casos, al usar las leyes de De Morgan para la negación de la conjunción. Es así como se concluye que al menos una de las dos personas no es italiana. Pero como puede ser inglesa o francesa, la decisión correcta es No se sabe, lo cual la hace diferente de los demás ítems en las cuales se puede contestar Sí o No, según la pregunta.

La discriminación de las respuestas de los estudiantes se presenta en la tabla 4.

En estos ítems, los aciertos de los estudiantes en el cuestionario final (25 aciertos de 36 o $69,44 \%$ ) se redujeron ostensiblemente con respecto al inicial (31 aciertos de 38 o $81,57 \%$ ) debido a que, sorprendentemente, sólo nueve de dieciocho $(50 \%)$ respondieron correctamente $b(i)$ en el cuestionario final. No encontramos explicación para el hecho de que aunque las dos ítems $b$ (i) tienen la misma estructura, haya una diferencia notable entre el número de aciertos del cuestionario inicial respecto del final. Por otro lado, debido a la complejidad del ítem $a$ (iv), es factible que los estudiantes hayan contestado correctamente No se sabe, no como consecuencia de un buen análisis, sino porque percibieron tal decisión como una forma de decir No sé cómo hacerlo. Lo anterior puede explicar por qué hubo mayor acierto para la decisión correcta en este ítem del cuestionario final.

\section{BREVE DISCUSIÓN DE RESULTADOS}

Para finalizar, hacemos algunas inferencias respecto a los efectos de la aplicación de las estrategias a lo largo del curso a partir de la comparación de resultados entre los cuestionarios inicial y final, presentados en las tablas $1,2,3$ y 4 .

Los resultados cuantitativos de respuestas categorizadas como deseables, en la categoría uno: «tipo de justificación» para los formatos Negación del antecedente, y $\mathrm{Ne}$ - gación del consecuente disminuyó entre un cuestionario y otro cuando se involucró una negación en la información proporcionada para las situaciones; consideramos que la causa de esta disminución puede deberse a que la mayoría de los teoremas y definiciones que se estudiaron para conformar el sistema axiomático en el curso del cual se obtuvo la información no tienen estos formatos y, por ello, a lo largo de la implementación no se hizo un tratamiento explícito de ese tipo de situación. En contraste, examinando esta misma categoría, cuando la información se ajusta a los demás formatos, las justificaciones deseables mejoraron ostensiblemente para situaciones en donde se afirma el consecuente y se mantuvo relativamente estable para la otra situación. Estos resultados concuerdan con la razón anteriormente expuesta.

En las situaciones en donde el formato de la información no corresponde a un esquema de razonamiento válido (negación del antecedente y afirmación del consecuente), el porcentaje de los elementos deseables que corresponden al uso de la condicional se incrementó, lo que significa que los estudiantes o bien identificaron que para estas situaciones, desde la lógica, no se puede deducir, de las premisas dadas, dato alguno para decidir Sí o No, o reconocieron el tipo de información adecuada para aplicar un esquema válido. El hecho de que se haya producido dicho incremento, lo podemos ver como un efecto positivo de la estrategia didáctica, implementada en el transcurso del curso, que solicita a los estudiantes estudiar si la información dada tiene o no las condiciones bajo las cuales es posible usar un esquema de inferencia.

Respecto de la segunda categoría «tipo de condicional», es importante resaltar que en la situación $b$ (ii) del cuestionario final hubo una disminución notoria en cuanto a los porcentajes de los elementos deseables. Esto posiblemente se debió a que se esperaba que, en situaciones como ésta (afirmación del antecedente), los alumnos hicieran un uso adecuado de la condicional; en este sentido, aunque todos los alumnos tomaron la decisión correcta, las justificaciones no fueron satisfactorias puesto que no se hacía explícita la premisa particular y, por ende, quedaron clasificados como ID. En resumen, creemos que los estudiantes consideraban obvia la justificación y por tanto no lo presentaban completamente.

Tabla 4

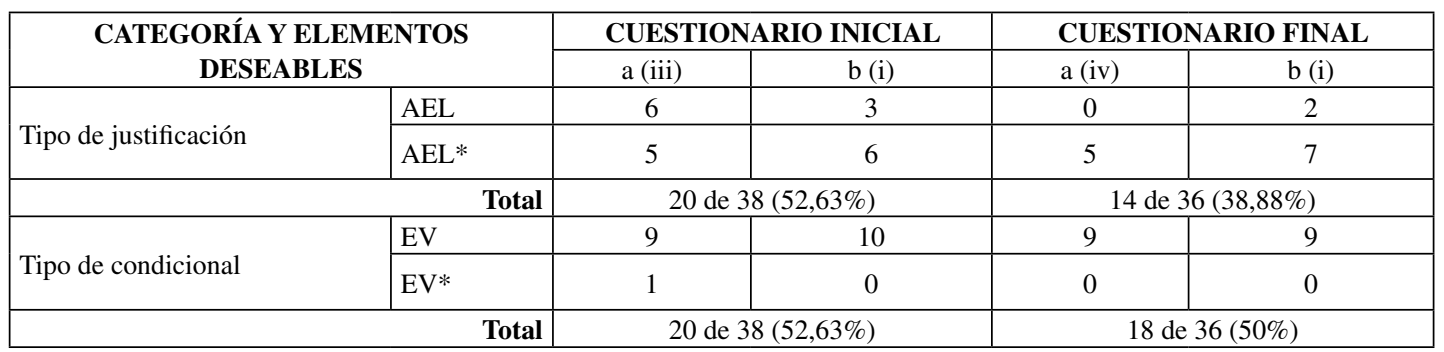

Frecuencias de justificaciones de los alumnos, en los elementos deseables de las dos categorías, para situaciones del formato negación del consecuente. 
Una estrategia que implementamos a lo largo del curso, consistente en solicitar a los estudiantes la reformulación de enunciados en la forma si-entonces y la identificación en ella, de la hipótesis y la tesis, parece tener un efecto significativamente positivo cuando la información corresponde a los primeros dos formatos: negación del antecedente y afirmación del consecuente; pero parece tener un efecto negativo aunque casi imperceptible para los otros dos formatos. Una posible interpretación de lo anterior es que los estudiantes ganaron un mayor grado de certeza para identificar las situaciones en las cuales está presente una condicional, pero no es posible hacer inferencia lógica alguna.

El resultado mencionado anteriormente respecto a las preguntas que atienden al formato negación del antecedente y al formato afirmación del consecuente puede ser indicio de un efecto positivo de las estrategias mencionadas, pues al finalizar el curso son más los estudiantes que reconocen cuándo no están dadas las condiciones para permitir una inferencia válida. Es sorprendente el cambio entre los porcentajes obtenidos cuando la información se presenta en el formato afirmación del antecedente. En el análisis de las producciones de los estudiantes a estas situaciones, específicamente la situación $b(i i)$ del cuestionario final, la definición de los elementos ID y EV de la segunda categoría no fue suficientemente específica para que nosotros pudiéramos llegar a un acuerdo al clasificar las correspondientes respuestas de los estudiantes. Esta situación tenía la información completa para hacer uso de MPP, y la pregunta acerca de la posibilidad de darse la condición expresada en el consecuente de la condicional, era directa. En el cuestionario inicial, la única situación del mismo formato es $b(i v)$ pero la pregunta es diferente porque no hace referencia a la misma característica del consecuente de la condicional. En ésta, además del uso de MPP, se necesita usar MTP.

\section{CONSIDERACIÓN FINAL}

Reconocemos entonces, con base en los comentarios expuestos anteriormente, que en términos generales las estrategias implementadas en el transcurso del curso tuvieron un efecto positivo en cuanto al tipo de justificación y al tipo de condicional en los estudiantes para situaciones sin un contexto matemático. En ese sentido, consideramos que para futuras investigaciones, el estudio puede centrarse en identificar los usos que dan los estudiantes a la condicional en situaciones con un contexto matemático específico.

\section{NOTAS}

1. Esta investigación titulada Aprendizaje de la demostración en geometría euclidiana con el apoyo de un programa de geometría dinámica se desarrolló con la financiación del Centro de Investigaciones de la Universidad Pedagógica Nacional (Bogotá, Colombia).

2. El grupo de investigación se denomina Aprendizaje y Enseñanza de la Geometría $(\notin \bullet G)$ y está adscrito al Departamento de Matemáticas de la Universidad Pedagógica Nacional.

3. En síntesis, para determinar si una proposición condicional es verdadera, es necesario entender la tabla de verdad, y es engorroso interpretar los casos donde el antecedente es falso. En la concepción matemática de la condicional se asume como verdadera una con antecedente falso para evitar hacer excepciones innecesarias. Por ejemplo, en la definición de diagonal de un polígono - una diagonal de un polígono es el segmento que une dos vértices no consecutivos del polígono- habría que exceptuar a los triángulos, lo cual complicaría la formulación de dicha definición.

4. Éste es un programa de formación de profesores para la educación básica y media (primaria y secundaria).

5. Al hacer el análisis de las producciones de los estudiantes recurrimos a designarlos no por un seudónimo sino por un código de la forma $E i$, con $i=1,2, \ldots, 19$.

6. El símbolo • después del símbolo $\rightarrow$ indica que no se pueden deducir propiedades de los elementos involucrados en la situación.

7. El esquema simbólico que expresa las situaciones es $[(c \rightarrow r) \wedge$ $c] \rightarrow r$.

8. Que se puede esquematizar $[(c \rightarrow r) \wedge c] \rightarrow r,[(r \vee t \vee d) \wedge r] \rightarrow \neg t$. 9. Que se puede esquematizar $[(c \rightarrow r) \wedge c] \rightarrow r$; al suponer que F5 es triangular se tiene $[(c \rightarrow r) \wedge \neg r] \rightarrow \neg c$ lo que lleva a contradicción. 


\section{REFERENCIAS BIBLIOGRÁFICAS}

DELOUSTAL-JORRAND, V. (2002). Implication and mathematical reasoning, en Cockburn, A. y Nardi, E. (eds.). Proceedings of the 26th Annual Conference of the International Group for the Psychology of Mathematics Education, 2, pp. 281-288. Norwich, UK: University of East Anglia.

DURAND-GUERRIER, V. (2003). Which notion of implication is the right one? From logical considerations to a didactic perspective. Educational Studies in Mathematics, 53, pp. 5-34.

HOYLES, C. y KÜCHEMANN, D. (2002). Students' understandings of logical implication. Educational Studies in Mathematics, 51, pp. 193-223.

LAUDIEN, R. (1999). Misunderstanding of if-then as if and only if, en Hitt, F. y Santos, M. (eds.). Proceedings of the 21 st Annual Meeting of the North American Chapter of the International Group for the Psychology of Mathematics Education, pp. 225-231. Columbus, OH: Eric Clearinghouse for Science Mathematics and Environmental Education.
PERRY, P., CAMARGO, L., SAMPER, C. y ROJAS, C. (2006). Actividad demostrativa en la formación inicial del profesor de matemáticas. Bogotá: Fondo Editorial de la Universidad Pedagógica Nacional.

SAMPER, C., PERRY, P., CAMARGO, L., MOLINA, Ó. y ECHEVERRY, A. (sometido a consideración). Conditional propositions: Problematic performances and didactic strategies.

SAMPER, C., PERRY, P., ECHEVERRY, A. y MOLINA, Ó. (2008). Estudio de la problemática asociada a la comprensión y el uso de la condicional en un curso de geometría plana (reporte de investigación DMA-06-12). Bogotá, Colombia: Universidad Pedagógica Nacional.

WASON, P.C. (1960). On the failure to eliminate hypothesis in a conceptual task. The Quarterly Journal of experimental Psychology, 12, pp. 129-140. 


\title{
Conditional statements: interpretation and use by pre-service mathematics teachers
}

\author{
Echeverry, Armando; Molina, Oscar; Samper, Carmen; Perry, Patricia y Camargo, Leonor \\ Universidad Pedagógica Nacional, Bogotá, Colombia \\ aecheverri@pedagogica.edu.co \\ ojmolina@pedagogica.edu.co \\ csamper@pedagogica.edu.co \\ pperry@yahoo.com.mx \\ lcamargo@pedagogica.edu.co
}

\section{Summary}

We report research findings related to the interpretation and use of conditional statements of a group of preservice mathematics teachers of a Euclidean geometry course. We analyzed the results of two questionnaires, with situations that did not have a mathematical context, applied it to the students, one at the beginning of the course and the other at the end. The changes would be generated by the implementation of didactic strategies throughout the semester that favored a comprehensive approach to the conditional statement from a mathematical point of view.

Our research has centered on didactic aspects related to the teaching and learning of proof in geometry. Since conditional statements are essential in the formulation and interpretation of theorems and postulates, and their structure is not easily adapted to the «if... then...» statements commonly used to argue in non-mathematical situations, we found it important to focus on this theme. There are two more reasons behind our decision: we found few studies of the comprehensions pre-service teachers have of conditional statements or how these develop throughout their schooling, and, teachers are not usually aware of the problems students have with respect to this issue.

Our research goal was to identify student problematic performances related to the use and interpretation of conditional statements, and design, apply and evaluate didactic strategies that could help students overcome the associated difficulties. Problematic performances are considered as actions that move away from the comprehension the community of mathematical discourse has of this mathematical object. The problematic performances found are: not identifying the hypothesis or thesis of a conditional statement completely; applying a postulate, theorem or definition without having the conditions of the corresponding hypothesis; conceiving the conditional in a restricted form by considering the antecedent as always true; and accepting the statement and its converse as equivalent.

We designed four didactic strategies. The purpose of Strategy A was to provide students with mechanisms to understand the structure of a conditional. Strategy B was meant to help students understand why using the converse or inverse statement does not always lead to a valid argument. With Strategy C, we provided experiences so that students could understand that a conditional statement could refer to dependence relations, identify hypothesis and thesis, and discriminate the relation between them. This strategy is closely related to the use of a dynamic geometry program throughout the semester as a source for information and as a semiotic mediator. The intention of Strategy D was to provide heuristic and technical elements to help students carry out deductive processes on their own.

The categories we used in order to analyze the students' productions led to the establishment of two descriptive typologies. The first category is type of justification; in the students' arguments to justify a decision we identified how the information given in the statements was used and whether the argument is based on a valid reasoning scheme or on the truth table of the conditional. With the other category, type of conditional, we identified the student's interpretation of the conditional without considering the relation that the argument has with the decision the student makes. That is, we were interested not only in identifying if the students used correct reasoning schemes but also determining whether the conditional was interpreted as equivalent to the converse, inverse or contrapositive. Specifically, to analyze students' productions with respect to the first category, we took into account the context of the situation presented, while the analysis with respect to the second category emphasized on the logical structure of the argument.

Our analysis shows that the strategies implemented during the course had a positive effect on our students with respect to type of justification and type of conditional, in the situations that are similar to the ones included in the questionnaires.

Key words: Proof, proving activity, use and interpretation of conditional statements, pre-service mathematics teachers. 